\title{
CLASSIFICATION OF SEMISIMPLE RANK ONE MONOIDS
}

\author{
BY
}

LEX E. RENNER

\begin{abstract}
Consider the classification problem for irreducible, normal, algebraic monoids with unit group $G$. We obtain complete results for the groups $\mathrm{Sl}_{2}(K) \times K^{*}$, $\mathrm{Gl}_{2}(K)$ and $\mathrm{PGl}_{2}(K) \times K^{*}$. If $G$ is one of these groups let $\mathscr{E}(G)$ denote the set of isomorphy types of normal, algebraic monoids with zero element and unit group $G$. Our main result establishes a canonical one-to-one correspondence $\mathscr{E}(G) \cong \mathbf{Q}^{+}$, where $\mathbf{Q}^{+}$is the set of positive rational numbers.

The classification is achieved in two steps. First, we construct a class of monoids from linear representations of $G$. That done, we show that any other $E$ must already be one of those constructed. To do this, we devise an extension principle analogous to the big cell construction of algebraic group theory. This yields a birational comparison morphism $\varphi: E_{r} \rightarrow E$, for some $r \in \mathbf{Q}^{+}$, which is ultimately an isomorphism because the monoid $E_{r} \in \mathscr{E}(G)$ is regular.

The relatively insignificant classification problem for normal monoids with group $G$ and no zero element is also solved. For each $G$ there is only one such $E$ with $G \varsubsetneqq E$.
\end{abstract}

Introduction. Let $K$ be an algebraically closed field. An algebraic monoid is an affine, algebraic variety $E$, defined over $K$, together with an associative morphism $m: E \times E \rightarrow E$ and a two-sided unit $1 \in E$ for $m$.

Several authors $[8,13,19]$ have studied algebraic monoids. Much of that work has been directed at the problem of relating monoid properties to properties of the unit group. In [14], for example, it is proved that any irreducible algebraic monoid with reductive unit group (e.g. $\left.\mathrm{Gl}_{n}(K)\right)$ is regular.

But no one has made a serious attempt at solving the classification problem: Given an irreducible reductive, algebraic group $G$, how does one ennumerate the essentially distinct, normal, irreducible algebraic monoids $E$, with unit group $G$ ?

That question is answered here completely in case $G$ is isomorphic to $\mathrm{Gl}_{2}(K)$, $\mathrm{Sl}_{2}(K) \times K^{*}$ or $\mathrm{PGl}_{2}(K) \times K^{*}$.

All of our efforts have been directed toward a classification in the spirit of modern algebraic group theory. What is ultimately hoped for is a one-to-one correspondence reductive, normal,
algebraic monoids $\leftrightarrow \begin{aligned} & \text { rational polyhedral } \\ & \text { root systems }\end{aligned}$

which generalizes the well-known classification for algebraic semisimple groups (see [2, Theorem 32]). All the findings of this paper support the hope that such a generalization is possible (see 4.5 and 5.3).

Received by the editors June 14, 1983.

1980 Mathematics Subject Classification. Primary 20M99.

Key words and phrases. Algebraic monoid, reductive, regular, $D$-monoid. 
What then is a rational polyhedral root system? Let $G$ be a reductive algebraic group and let $T \subseteq G$ be a maximal torus. From the adjoint representation Ad: $T \rightarrow \operatorname{Aut}(\&)$ ) (see $[2, \S 10.3]$ ) we obtain the root system $(X(T) \otimes \mathbf{R}, \Phi)$ of $G$, where $X(T)$ is the set of characters of $T$ and $\Phi \subseteq X(T)$ is the set of (nonzero) weights of $T$ on $B$.

If $G$ is also the unit group of an algebraic monoid $E$, then the Zariski closure $\bar{T}$ of $T$ in $E$ is a torus imbedding in the sense of [ 3 and 4]. But $\bar{T}$ has a richer structure relating to the inclusion of $T$ in $G$ and the associated Weyl group action.

Let $X(\bar{T})=\operatorname{Hom}(\bar{T}, K)$ be the set of monoid morphisms and note that $X(\bar{T}) \subseteq$ $X(T)$ by restriction. We then have, by definition, the polyhedral root system $(X(\bar{T}), \Phi)$ of $E$. (This is an introductory formulation.) The picture for $G=\mathrm{Gl}_{2}(K)$ and $E=M_{22}$ is as follows:
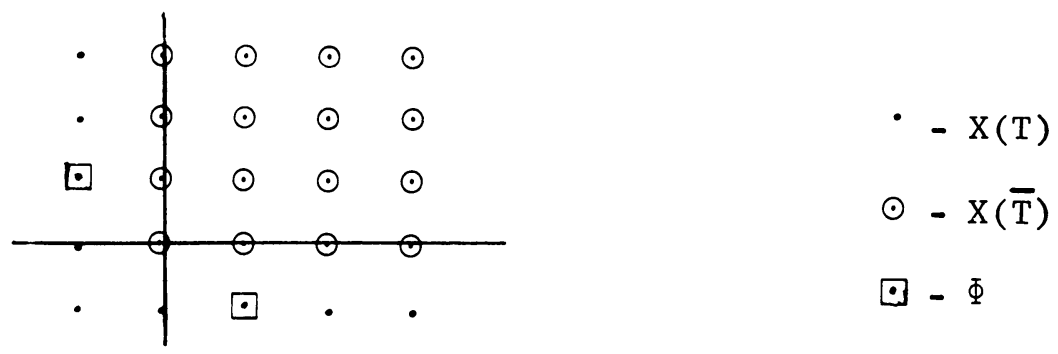

In this case

$$
\begin{gathered}
T=\left\{\left[\begin{array}{ll}
\alpha & 0 \\
0 & \beta
\end{array}\right] \mid \alpha \beta \neq 0\right\}, \quad \bar{T}=\left\{\left[\begin{array}{ll}
\alpha & 0 \\
0 & \beta
\end{array}\right]\right\}, \\
X(T) \cong \mathbf{Z} \oplus \mathbf{Z}, \quad \text { generated by }\left[\begin{array}{ll}
\alpha & 0 \\
0 & \beta
\end{array}\right] \mapsto \alpha \text { and }\left[\begin{array}{ll}
\alpha & 0 \\
0 & \beta
\end{array}\right] \mapsto \beta, \\
X(\bar{T})=\{(a, b) \in X(T) \mid a, b \geqslant 0\} \cong \mathbf{N} \oplus \mathbf{N}, \\
\Phi=\{(1,-1),(-1,1)\} \subseteq X(T) .
\end{gathered}
$$

With this in mind we state the main results.

THEOREM. Suppose $G$ is one of the groups $\mathrm{Gl}_{2}(K), \mathrm{Sl}_{2}(K) \times K^{*}$ or $\mathrm{PGl}_{2}(K) \times K^{*}$. Let $\mathscr{E}(G)$ denote the set of isomorphy classes of irreducible, normal, algebraic monoids with 0 and unit group $G$. Then there is a canonical one-to-one correspondence $\mathscr{E}(G) \cong \mathbf{Q}^{+}$, where $\mathbf{Q}^{+}$denotes the set of positive rational numbers.

Furthermore, each $E \in \mathscr{E}(G)$ is uniquely determined by its polyhedral root system $(X(\bar{T}), \Phi)$.

The proof is achieved in two stages. First we construct monoids from linear representations of $G$. Then we prove that these monoids exhaust the possibilities. (The less significant case of monoids without 0 is also treated.) A major consequence of our approach is a complete list of these polyhedral root systems. For simplicity let $G=\mathrm{Sl}_{2}(K) \times K^{*}$. 
EXAMPLE. $E=E(2 / 3) \in \mathscr{E}(G)$

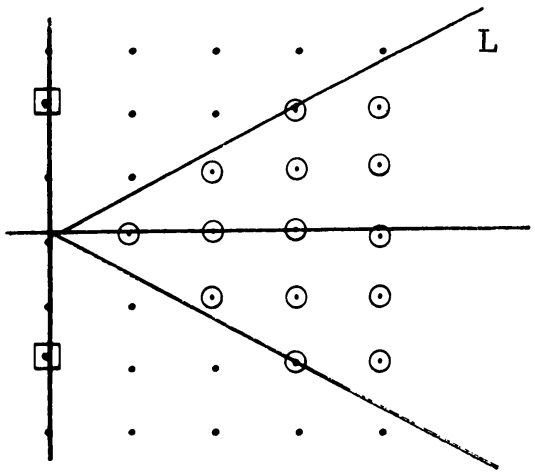

$$
\begin{gathered}
\cdot-\mathrm{X}(\mathrm{T}) \\
\odot-\mathrm{X}(\overline{\mathrm{T}}) \\
\square-\Phi \\
2 / 3=\operatorname{slope}(\mathrm{L})
\end{gathered}
$$

1. Terminology and background. Let $K$ be an algebraically closed field. An affine algebraic monoid $E$ is an affine algebraic variety defined over $K$, together with an associative morphism $m: E \times E \rightarrow E$ and a two-sided unit $1 \in E$ for $m$. See [8, 11, 9 and 13].

If $E$ is an affine algebraic monoid then $G(E)=\left\{x \in E \mid x^{-1} \in E\right\}$ is an affine algebraic group [5, Corollary 1.2] and $G(E) \subseteq E$ is an open subset in the Zariski topology [loc. cit.]. Furthermore, $\operatorname{dim}(E \backslash G(E))=\operatorname{dim} E-1$ if $E$ is irreducible [10]. $E^{0}$, the irreducible component of 1 , is the unique, maximal, closed, irreducible submonoid of $E$ [loc. cit.]. $I(E)=\left\{e \in E \mid e^{2}=e\right\}$ is the set of idempotents of $E$.

A $D$-monoid is an irreducible, algebraic monoid $Z$ with $G(Z) \cong G_{m} \times \cdots \times G_{m}$, where $G_{m} \cong K^{*}$ is the one dimensional multiplicative group. $X(Z)=\operatorname{Hom}\left(Z, A^{1}\right)$ is the set of characters of $Z$. If $E$ is an affine algebraic monoid, a maximal D-submonoid of $E$ is a $D$-monoid which is maximal among submonoids of $E$ with this property. A $D$-monoid $Z$ is determined to within an isomorphism by the finitely generated commutative monoid $X(Z)$ [7, Theorem 2.6].

$E$ is regular if for each $x \in E$ there exists $a \in E$ such that $x a x=x$. By the results of [12], an irreducible, algebraic monoid $E$ is regular if and only if, for each $x \in E$ there exists $g \in G(E)$ and $e \in I(E)$ such that $g e=x$.

An irreducible algebraic monoid $E$ is reductive if $G(E)$ is a reductive algebraic group [2, Chapter 10]. In [11 and 14] it is proved that any reductive monoid is regular. This is perhaps our most fundamental finding on the subject.

An irreducible algebraic variety is normal if $K[X]$, the coordinate ring of $X$, is integrally closed in its field of fractions. It is a simple matter, using the universal property of integral closure [17, p. 113], to see that the normalization $\eta: \tilde{E} \rightarrow E$ of an algebraic monoid $E$ is a normal algebraic monoid and $\eta$ is a finite birational morphism of algebraic monoids. The reader should note that monoids, unlike groups, have singularities in general. For the simplest example, take the cusp $E=\left\{(x, y) \in K \times K \mid x^{2}=y^{3}\right\}$ with coordinatewise multiplication.

If $X$ is a monoid (usually $X(\bar{T})$ ) and $x_{1}, x_{2}, \ldots, x_{n} \in X$, then $\left\langle x_{1}, \ldots, x_{n}\right\rangle$ denotes the submonoid of $X$ generated by $x_{1}, \ldots, x_{n}$.

An irreducible, algebraic monoid $E$ is solvable if $G(E)$ is a solvable, algebraic group [2, Chapter 7]; or equivalently, if there exists a faithful, linear representation 
$\rho: E \rightarrow \operatorname{End}_{K}(V)$ such that $\rho(E)$ consists of upper-triangular matrices (see [2, Theorem 17.6] and [5, Theorem 1.1]).

If $e \in I(E)$ then $e E e$ is a closed subset of $E$ and is, therefore, an algebraic monoid with identity $e$. We let $G(e) \subseteq e E e$ denote the unit group of $e E e$.

If $x \in E$ then $\mathrm{Cl}(x)$ denotes the $G(E)$-conjugacy class of $x$ in $E$.

In this paper we are primarily concerned with monoids relating to the groups $\mathrm{Gl}_{2}(K), \mathrm{Sl}_{2}(K) \times K^{*}$ and $\mathrm{PGl}_{2}(K) \times K^{*}$. These monoids are all constructed from representations of $\mathrm{Sl}_{2}(K) \times K^{*}$. We use the symmetric power representations induced from the canonical two dimensional representation $\mathrm{Sl}_{2}(K) \subseteq \mathrm{Gl}_{2}(K)$. One considers these all at once by extending this representation to a group of automorphisms of the graded symmetric algebra $S^{*}\left(K^{2}\right)=\oplus_{k \geqslant 0} S^{k}\left(K^{2}\right) \cong K[X, Y]$.

We recall a number of basic principles from algebraic geometry.

If $X$ and $Y$ are irreducible algebraic varieties and $\varphi: X \rightarrow Y$ is a morphism, then $\varphi$ is dominant if $\varphi(X) \subseteq Y$ is dense in the Zariski topology. For such a morphism, the dimension theorem states that $r=\operatorname{dim} X-\operatorname{dim} Y \geqslant 0$ [17, p. 60]. Further, if $y \in \varphi(X)$ then $\operatorname{dim} Z \geqslant r$ for each irreducible component $Z$ of $\varphi^{-1}(y)$ [loc. cit.]. We shall refer to this result as the dimension theorem.

A morphism $\varphi: X \rightarrow Y$ of irreducible varieties is birational if for some open set $U \subseteq X,\left.\varphi\right|_{U}: U \rightarrow Y$ is an isomorphism of $U$ onto some open subset of $Y$. Zariski's main theorem (ZMT) asserts that if $Y$ is normal, $\varphi$ is birational and $\varphi^{-1}(y)$ is either empty or finite for each $y \in Y$, then $\varphi$ is an isomorphism of $X$ onto its image $\varphi(X) \subseteq Y$. Further, $\varphi(X) \subseteq Y$ is open. In practice, ZMT is often very easy to apply. Typically, (see 3.2 and 5.4) one constructs a birational morphism $\varphi: X \rightarrow Y$ which is, ultimately, an isomorphism. To verify this, it suffices, assuming $Y$ is normal, to check that $\varphi$ is onto with finite fibres. In the theory of algebraic grops one may often avoid this relatively powerful technique by invoking a homogeneity argument as in Proposition 28.5 of [2]. However, no such homogeneity is available in a monoid.

2. Two dimensional regular monoids. This section is devoted to a discussion of irreducible, algebraic monoids of the prototype

$$
E=\left\{\left[\begin{array}{ll}
1 & b \\
0 & a
\end{array}\right] \in M_{22}(K)\right\}
$$

Our motivation here is to obtain sufficient information about irreducible, normal, monoids $E$ with unit group $\mathrm{Gl}_{2}(K)$ to construct an open subset $U \subseteq E$ analogous to

$$
U=\left\{\left[\begin{array}{ll}
u & v \\
w & y
\end{array}\right] \in M_{22}(K) \mid y \neq 0\right\} .
$$

The siginificant property of $U$ is that the morphism

$$
\varphi:\left\{\left[\begin{array}{ll}
1 & b \\
0 & 1
\end{array}\right]\right\} \times\left\{\left[\begin{array}{ll}
u & 0 \\
0 & y
\end{array}\right] \mid y \neq 0\right\} \times\left\{\left[\begin{array}{ll}
1 & 0 \\
a & 1
\end{array}\right]\right\} \rightarrow U
$$

defined by

$$
\begin{aligned}
\varphi\left(\left[\begin{array}{ll}
1 & b \\
0 & 1
\end{array}\right],\left[\begin{array}{ll}
u & 0 \\
0 & y
\end{array}\right],\left[\begin{array}{ll}
1 & 0 \\
a & 1
\end{array}\right]\right) & =\left[\begin{array}{ll}
1 & b \\
0 & 1
\end{array}\right]\left[\begin{array}{ll}
u & 0 \\
0 & y
\end{array}\right]\left[\begin{array}{ll}
1 & 0 \\
a & 1
\end{array}\right] \\
& =\left[\begin{array}{cc}
u+a b y & b y \\
a y & y
\end{array}\right]
\end{aligned}
$$

is an isomorphism of varieties. 
Once we have such a subset the task of constructing morphisms from the normal monoid $E$ is greatly simplified (see 3.1 ).

Let $E$ be a two dimensional irreducible algebraic monoid and let $G=G(E)$. By [2, Ex. \#4, p. 137], $G$ is a solvable algebraic group and hence $G$ is either

(i) unipotent or

(ii) $G_{m} \times G_{m}$ or

(iii) an extension of $G_{a}$ by $G_{m}$.

We may rule out (i), since by [19], a necessary and sufficient condition for $G \subsetneq E$ is that $X(G)=\operatorname{Hom}\left(G, G_{m}\right) \neq\{e\}$.

2.1. Proposition. Let $E$ be as above and assume that $G \subsetneq E$. Assume also that $E$ is not a D-monoid (i.e. G satisfies (iii) above). Then the following are equivalent.

(i) $E$ is not commutative and does not have a zero.

(ii) $E=G \cup \mathrm{Cl}(e)$ where $e \in E$ is any idempotent $e \neq 1$.

In particular, $E$ is a regular monoid.

Proof. By our assumptions $G$ is an extension of $G_{a}$ by $G_{m}$.

By [5, Theorem 1.1] we can embed $j: E \rightarrow$ End $_{K}(V)$ for some $V$. But then $\rho=\operatorname{det} \circ j: E \rightarrow K$ is a surjective morphism since $\rho(1)=1, \rho(E \backslash G)=0$ and $\rho(E) \subseteq K$ is an irreducible subset. We use $\rho$ in the proof of (ii) $\Rightarrow$ (i). (i) $\Rightarrow$ (ii). By [5, Corollary 1.4] there exists $e=e^{2} \in E, e \neq 1$ and by [7, Corollary 1.6] $e \in \overline{K^{*}}$ where $K^{*} \subseteq G$ is some one parameter subgroup. Thus, $e t=t e=e$ for all $t \in K^{*}$. Since $G$ is not commutative $K^{*} \subseteq G$ is not central. But then by [2, Corollary 16.3] $K^{*}$ is not normal in $G$ either. We thus conclude that $Z=\bigcup_{g \in C} g K^{*} g^{-1} \subseteq G$ is dense for dimensional reasons. But now $\operatorname{dim} \mathrm{Cl}(e)=1$ since otherwise $g e=e g$ for all $g \in G$ and this would force $e \in \overline{g K^{*} g^{-1}}$ for all $g \in G$ and thus $e t=t e=e$ for all $t \in Z$. But this contradicts the facts that $Z \subseteq E$ is dense and $e$ is not the zero element.

Now let $V \subseteq E \backslash G$ be an irreducible component. Then $E \cdot V \cdot E \subseteq V$ since $E(E \backslash G) E \subseteq E \backslash G$ and $E$ is connected. Hence, there exists $f=f^{2} \in V$ [5, Corollary 1.4]. But then $f \in \mathrm{Cl}(e)$ since by [2, Theorem 19.3] the one parameter subgroup of $e$ is conjugate to the one parameter subgroup of $f$. Hence, $\mathrm{Cl}(e) \subseteq V$, and by dimension count $\mathrm{Cl}(e)=V$ since $V$ is irreducible. Thus, $E=G \cup \mathrm{Cl}(e)$. (ii) $\Rightarrow$ (i) It suffices to show that if $E$ has a zero then $E=G \cup \mathrm{Cl}(e)$ is impossible. Now $\rho=\operatorname{det} \circ j: E \rightarrow K$ satisfies $\rho^{-1}(0)=E \backslash G=\{e\}$ by assumption. But by the dimension theorem we must have $\operatorname{dim} E \backslash G \geqslant 1$. This contradiction concludes the proof.

2.2. Corollary. Suppose $E$ is as in 2.1. Then either

(i) $e E=\mathrm{Cl}(e)$ and $E e=\{e\}$ or

(ii) $E e=\mathrm{Cl}(e)$ and $e E=\{e\}$.

Proof. If $e, f \in \mathrm{Cl}(e)$ then $e f=f e$ implies $e=f$ since also, ef $\in \mathrm{Cl}(e)$ (we can think of $E \subseteq \operatorname{End}(V)$ for some $V$ ). Thus, $e \cdot E \cdot e=\{e\}$. Since $e$ is not the zero element and $\operatorname{dim} \mathrm{Cl}(e)=1$, either $e E=\mathrm{Cl}(e)$ or $E e=\mathrm{Cl}(e)$. The conclusion thus follows. 
Assume now that $e E=\mathrm{Cl}(e)$ and $E e=\{e\}$ (the other case is similar). The example to keep in mind is the set of two-by-two upper-triangular matrices $\left(a_{i j}\right)$ with $a_{11}=1$.

Let $K^{*} \subseteq G \subseteq G(E)$ be the one parameter subgroup with $e \in \overline{K^{*}}$. From the structure theory for solvable groups [loc. cit.] the morphism of varieties

$$
m: K^{*} \times G_{a} \rightarrow G, \quad(t, u) \mapsto t \cdot u
$$

is an isomorphism. Further $m$ extends to a morphism

$$
m: K \times G_{a} \rightarrow E, \quad(0, u) \mapsto e \cdot u .
$$

$m$ is surjective since $E=G \cup e E$, but $e E=e G_{a}$ since the dimensions are the same and $e G_{a} \subseteq e E$ is closed.

2.3. Proposition. Let $E$ be as above and let $e \in E \backslash G$ with one parameter subgroup $K^{*} \subseteq G, e \in \overline{K^{*}}$. Then the action $(t, x) \rightarrow t^{-1} x t$ of $K^{*}$ on $E$ extends to an action of $K$ on $E$.

Proof. We write $\lim _{t \rightarrow 0} t^{-1} x t=y$ if the morphism $\varphi: K^{*} \rightarrow E, t \rightarrow t^{-1} x t$, can be extended to $K$, with $\varphi(0)=y . y$ is then unique because $K^{*} \subseteq K$ is dense.

Since $G$ is not commutative, the morphism $\rho: K^{*} \rightarrow \operatorname{Aut}\left(G_{a}\right), \rho(t)(u)=t^{-1} u t$, is not the trivial one. Thus, there is an isomorphism $G_{a} \cong \mathbf{A}^{1}$ such that the resulting action $\rho$ is defined on $\mathbf{A}^{1}$ by

$$
\rho(t)(u)=\left\{\begin{array}{l}
t \cdot a \text { or } \\
t^{-1} \cdot a
\end{array}\right.
$$

for all $t \in K^{*}$. Hence, for all $t \in K^{*}$, either

$$
\lim _{t \rightarrow 0} t u t^{-1}=1 \quad \text { or } \quad \lim _{t \rightarrow 0} t^{-1} u t=1 \text {. }
$$

We rule out the first case.

Suppose then, that

$$
\lim _{t \rightarrow 0} t u t^{-1}=1
$$

Then

$$
\lim _{t \rightarrow 0} t_{e u t^{-1}}=\lim _{t \rightarrow 0} e t u t^{-1}=e \lim _{t \rightarrow 0} t u t^{-1}=e .
$$

But

$$
\lim _{t \rightarrow 0} t^{-1} e u t=\lim _{t \rightarrow 0} e u t=e u e=e
$$

since by definition $e \in \overline{K^{*}}$. Thus, we obtain a nonconstant morphism (choosing $u$ such that $e u \neq e$, as in 2.2(i)).

$$
\begin{aligned}
\mathbf{P}^{1} & \rightarrow E, \\
{[t: 1] } & \mapsto \text { teut }^{-1}, \\
{[1: t] } & \mapsto t^{-1} \text { eut } .
\end{aligned}
$$

But this contradicts a well-known property of $\mathbf{P}^{1}$; that it possesses no nonconstant functions to any affine variety. 
Thus, we must have

$$
\lim _{t \rightarrow 0} t^{-1} u t=1 \quad \text { for all } u \in G_{a} .
$$

In particular, $\lim _{t \rightarrow 0} t^{-1} x t$ exists in $E$ for all $x \in E$ (using the surjective morphism of (1)).

It now follows easily that the action can be extended as claimed.

We now record several results about monoids $E$ with 0 and unit group $\mathrm{Gl}_{2}(K)$, $\mathrm{Sl}_{2}(K) \times K^{*}$ or $\mathrm{PGl}_{2}(K) \times K^{*}$. These will be applied in $\S 4$.

2.4. Proposition. Let $E$ be as above and let $e \in E$ be an idempotent $e \neq 0$ or 1 . Let

$$
R(e)=\{g \in G \mid e g=e g e\} \quad \text { and } L(e)=\{g \in G \mid g e=e g e\} .
$$

Then $R(e)$ and $L(e)$ are opposite Borel subgroups.

Proof. See [9, Theorem 4.5].

\subsection{Corollary. Let $E$ and $e$ be as in 2.4. Then}

$$
\operatorname{dim} E \cdot e=\operatorname{dim} e \cdot E=2 .
$$

Proof. By [12, Theorem 1], $E \cdot e \subseteq \overline{R(e)}$ so $\operatorname{dim} E \cdot e<3$. Since $R(e) \subsetneq G$ there exists $u \in G$ unipotent such that $u e \neq e$. But then $\operatorname{dim} E \cdot e=\operatorname{dim} G \cdot e \geqslant 2$. Thus, $\operatorname{dim} E \cdot e=2$. The other case is similar.

3. Big cell. In the study of semisimple groups, Chevalley introduced the notion of a "big cell" which he then used to construct linear representatons in sufficient numbers. In the more modest classification problem, solved here, a similar procedure is needed to construct certain morphisms of algebraic monoids.

We shall need the following result from commutative algebra. For more details see [1, §5, Lemma 1]. This basic result also has important consequences in invariant theory.

3.1 Codimension $\geqslant 2$ condition. Suppose $X$ is an irreducible, normal, affine variety and $U \subseteq X$ is an open subset such that $\operatorname{dim}(X \backslash U) \leqslant \operatorname{dim} X-2$. Then any morphism $f: U \rightarrow Y$ from $U$ to an affine variety $Y$ can be extended uniquely from $X$ to $Y$.

We fix notation and summarize some results about algebraic monoids.

$E$ is an irreducible, normal, algebraic monoid with 0 and unit group $\mathrm{Gl}_{2}(K)$, $\mathrm{Sl}_{2}(K) \times K^{*}$ or $\mathrm{PGl}_{2}(K) \times K^{*} . \bar{T} \subseteq E$ is the closure in $E$ of a maximal torus $T$ of $G$. Let $e \in \bar{T}$ be an idempotent $\neq 0$ or 1 and let $B$ and $B^{-}$be the Borel subgroups of $G$ containing $T$. Let $U$ and $U^{-}$be the unipotent radicals of $B$ and $B^{-}$, respectively.

From [6, Theorem 3.20] it follows that $I(\bar{T})=\{1, e, f, 0\}$ with ef $=0$.

$\bar{T}_{e}=\bar{T} \backslash f \cdot \bar{T}$ is the unique open submonoid of $\bar{T}$ with $I\left(\bar{T}_{e}\right)=\{1, e\}$. Further, $\bar{T}_{e}$ is affine [15] and $\bar{T}_{e} \backslash T=Z \cdot e$ where $Z \subseteq G$ is the identity component of the center of $G$.

Let $K^{*} \subseteq T$ be the one parameter subgroup of $T$ with $e \in \overline{K^{*}} . e$ is an element of both $\overline{K^{*} \cdot U}$ and $\overline{K^{*} \cdot U^{-}}$, which by 2.1 , are regular monoids.

By 2.2 and 2.5 we may assume

$$
\bar{B} \cdot e=E e \text { and } e \cdot \bar{B}^{-}=e E .
$$


Consider the morphism of varieties

$$
m: U \times \bar{T}_{e} \times U^{-} \rightarrow E, \quad m(x, y, z)=x \cdot y \cdot z .
$$

By [2, Proposition 28.5] $m$ is birational. To show that $m$ is finite to one, it suffices to show that $m^{-1}(e)$ is finite, since by (2) above and 2.2, $U \times e T \times U^{-}$is an orbit under the group action

$$
\begin{gathered}
U \times Z \times U^{-} \times\left(U \times e T \times U^{-}\right) \rightarrow\left(U \times e T \times U^{-}\right), \\
(u, t, v) *(x, y, z)=\left(u x, t y, z v^{-1}\right) .
\end{gathered}
$$

Suppose then that $x y z=e, x \in U, y=e t, t \in Z$ and $z \in U^{-}$. Then etz $=x^{-1} e$ and thus, $x^{-1} e$ commutes with $e$. But then 2.2 applied to $\overline{K^{*} \cdot U}$ implies that $x^{-1} e=e$. Similarly $e z=e$. Thus, $t e=e$ as well. But,

$$
\left\{(x, t, z) \in U \times Z \times U^{-} \mid x e=t e=e z=e\right\}
$$

is finite, since $\operatorname{dim}(U e)=\operatorname{dim}(Z e)=\operatorname{dim}\left(e \cdot U^{-}\right)=1$.

3.2. PRoposition. $m$ : $U \times \bar{T}_{e} \times U^{-} \rightarrow E$ is an open embedding.

Proof. $m$ is finite to one and birational. Since $E$ is normal by definition, $m$ is an open embedding by ZMT.

3.3. Corollary (Extension Principle). Let $E$ be as above and let $E^{\prime}$ be any affine, algebraic monoid. Suppose we are given morphisms

$$
\rho: G(E) \rightarrow E^{\prime} \text { and } \alpha: \bar{T}_{e} \rightarrow E^{\prime}
$$

of algebraic monoids such that $\left.\rho\right|_{T}=\left.\alpha\right|_{T}$. Then there exists a unique morphism $\beta$ : $E \rightarrow E^{\prime}$ extending both $\rho$ and $\alpha$.

Proof. Let $V=U \times \bar{T}_{e} \times U^{-} \subseteq E$ be as in 3.2. Define $\beta^{\prime}: V \rightarrow E^{\prime}, \beta^{\prime}(x, y, z)=$ $\rho(x) \alpha(y) \rho(z)$. So $\beta^{\prime}$ agrees with $\rho$ on $G=G(E)$ and with $\alpha$ on $\bar{T}_{e}$. Thus, there exists $\beta^{\prime \prime}: W \rightarrow E^{\prime}$ extending both $\rho$ and $\beta^{\prime}$, where $W=V \cup G$.

By [14], $E$ is a regular monoid, so for any $x \in(E \backslash G) \backslash\{0\}$ we can find $g, h \in G$ such that $g x h=e$ or $f$. But $e$ and $f$ are conjugate under the Weyl group.

Thus, $(E \backslash G) \backslash\{0\}=G \cdot e \cdot G$ is an irreducible variety. But, $E \backslash W \subsetneq E \backslash G$, and so $\operatorname{dim}(E \backslash W) \leqslant \operatorname{dim} E-2$. Hence, by 3.1 , the morphism $\beta^{\prime \prime}: W \rightarrow E^{\prime}$ extends uniquely to $\beta$ : $E \rightarrow E^{\prime}$. But then $\beta$ is a morphism of monoids because $G \subseteq E$ is dense and $\left.\beta\right|_{G}=\rho$.

4. Existence. The purpose of this section is to construct, for each of the groups $G=\mathrm{Gl}_{2}(K), \mathrm{Sl}_{2}(K) \times K^{*}$ and $\mathrm{PGl}_{2}(K) \times K^{*}$, all irreducible, normal, algebraic monoids $E$ with unit group $G$. At the outset there are two basic types to consider, depending on whether the monoid has a zero. Throughout this section we are concerned only with the monoids $E$ with zero. The other case, which is much simpler, will be treated in $\S 5$.

The basic strategy of the construction is straightforward and is summarized here.

Given an irreducible, normal, algebraic monoid $E$, there exists a representation $\rho$ : $G(E) \rightarrow \mathrm{Gl}(V)$ such that $E \cong \overline{\rho(G(E))} \subseteq \operatorname{End}(V)$ (Zariski closure). Furthermore, by the results of $\S 3$, it will turn out that each $E$ is determined by $\bar{T}$, the closure in $E$ 
of some maximal torus $T$ of $G(4.5)$. Thus, to construct the possible monoids it suffices to enumerate the theoretically possible $\bar{T}$ 's and then show that these all actually occur using the representations of $G$ with the appropriate weights. For example, the monoid $E$ with group $\operatorname{PGl}_{2}(K) \times K^{*}$ and $\bar{T}=\left\{(a, b, c) \in K^{3} \mid b^{2}=\right.$ $a c$ \} comes from the binary quadratic representation of $\operatorname{PGl}_{2}(K)$ (i.e.: $S^{2}\left(K^{2}\right) \cong K$. $\left.X^{2} \oplus K \cdot X Y \oplus K \cdot Y^{2} \subseteq K[X, Y]\right)$.

For the remainder of this section we let $G=\mathrm{Sl}_{2}(K) \times K^{*}$. The other two groups are similar and will be discussed briefly at the end of the section.

We fix notation.

$E$ is a normal algebraic monoid with 0 and unit group $G$.

$\bar{T}$ is the closure in $E$ of a maximal torus $T$ of $G$. We assume

$\bar{T}$ is a normal variety. This will be proved later (5.2).

$X(\bar{T})=\operatorname{Hom}\left(\bar{T}, \mathbf{A}^{1}\right) \subseteq X(T)$.

$X(T)=\operatorname{Hom}\left(T, G_{m}\right) \cong \mathbf{Z} \oplus \mathbf{Z}$.

$\Phi=\{\alpha,-\alpha\} \subseteq X(T)$ the roots, $\tau: X(\bar{T}) \rightarrow X(\bar{T})$, the action of the nontrivial element $\tau \in N_{G}(T) / T$.

Since $G=K^{*} \times \mathrm{Sl}_{2}(K)$ we get $T=K^{*} \times H$ where $H \subseteq \mathrm{Sl}_{2}(K)$ is conjugate to

$$
\left\{\left[\begin{array}{cc}
t & 0 \\
0 & t^{-1}
\end{array}\right] \mid t \in K^{*}\right\}
$$

$\tau$ acts on $T$ by $\tau(x, y)=\left(x, y^{-1}\right)$, and thus, $\tau$ acts on $X(T) \cong \mathbf{Z} \oplus \mathbf{Z}$ by $\tau(m, n)=$ $(m,-n)$. Note also that $\Phi=\{(0,2),(0,-2)\}$.

As $\bar{T}$ is a normal torus embedding, there is a rational polyhedral cone $\sigma \subseteq X(T)$ $\otimes \mathbf{Q}$ such that $X(\bar{T})=\sigma \cap X(T)$ [3, p. 7-8]. (We are actually using the dual of the construction used in [loc. cit.]).

Since $\bar{T}$ has a zero, $\sigma$ is the convex hull of two lines emanating from the origin.

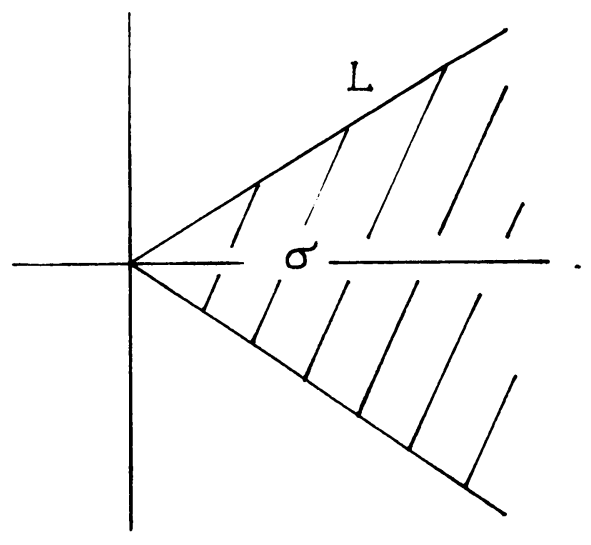

Further, $\tau(\sigma)=\sigma$ since $\sigma$ is the convex hull of $X(\bar{T})$ [loc. cit.].

The rationality of $\sigma$ means that the slope of $L$ is a rational number.

By [6, Theorem 3.20] $I \bar{T}=\{1, e, f, 0\}$ and by $[9$, Theorem 3.10] $\tau(e)=f$. 
By [4, p. 6] there exists $F_{1}, F_{2} \in X(\bar{T})$ such that $k \cdot x \in\left\langle F_{1}, F_{2}\right\rangle$ for all $x \in X(\bar{T})$ and some integer $k>0 .\left\{F_{1}, F_{2}\right\}$ is unique with the property that $F_{i}, i=1,2$, is not a linear combination with positive rational coefficients of any other elements of $X(\bar{T}) . F_{1}$ and $F_{2}$ are called the fundamental generators of $X(\bar{T})$. We can identify $\left\{F_{1}, F_{2}\right\}$ on the above illustration as the integral points on the boundary of $\sigma$ with minimal distance from the origin. Clearly, $\tau\left(F_{1}\right)=F_{2}$, so let $F_{1}=(m, n), F_{2}=$ $(m,-n)$. It is now clear that

$$
X(\bar{T})=\{(a, b) \in X(T) \cong \mathbf{Z} \oplus \mathbf{Z}|| a / b \mid \leqslant m / n\} \cup\{(0,0)\} .
$$

\section{Notation.}

$$
\begin{aligned}
& X(r):=\left\{(a, b) \in \mathbf{Z}^{2}|| a / b \mid \leqslant r\right\} \cup\{(0,0)\}, \quad \text { where } r \in \mathbf{Q}^{+}, \\
& \bar{T}(r):=\bar{T} \text { if } X(\bar{T}) \cong X(r) .
\end{aligned}
$$

We now proceed to the construction. The first step is to create a monoid with given $X(r), r \in \mathbf{Q}^{+}$.

For $(k, l) \in X(r)$ define $\rho_{k, l}: \mathrm{Sl}_{2}(K) \times K^{*} \rightarrow \mathrm{Gl}_{l+1}(K)$ by the formula $\rho_{k, l}(x, t)=S^{l+1}(x) \cdot x_{k}(t)$, where $S^{l+1}$ is the $l+1$ - symmetric power representation and

$$
x_{k}(t)=\left[\begin{array}{cc}
t^{k} & 0 \\
0 & t^{k}
\end{array}\right]
$$

The weights of $\left.\rho_{k, l}\right|_{T}$ on $K^{l+1}$ are

$$
W(k, l)=\{(k, l),(k, l-2), \ldots,(k,-l+2),(k,-l)\} .
$$

Further, $W(k, l) \subseteq X(r)$.

By Gordon's lemma [3, p. 7], $X(r)$ is a finitely generated monoid, so choose $\left\{\left(m_{i}, n_{i}\right)\right\} \subseteq X(r)$ which generate it. Define

$$
\rho=\bigoplus_{i=1}^{s} \rho_{m_{i}, n_{i}}: \mathrm{Sl}_{2}(K) \times K^{*} \rightarrow \mathrm{Gl}_{t}(K), \quad \text { where } t=\sum_{i=1}^{s} n_{i}+s .
$$

4.1. Lemma. Let $G$ be a connected algebraic group with maximal torus $T$ and let $\rho$ : $G \rightarrow \mathrm{Gl}(V)$ be a representation. Let $\Phi(\rho) \subseteq X(T)$ be the set of weights of $\rho$. Then $\overline{\rho(T)} \subseteq \operatorname{End}(V)$ is a closed submonoid with $X(\overline{\rho(T)})=\langle\Phi(\rho)\rangle$ (the submonoid generated by $\Phi(\rho))$.

Proof. Since $T$ is, by definition, a $D$-group, we may assume by [2, Proposition 15.4] that $\rho(T) \subseteq D(V)$, the set of diagonal matrices. Thus we can reduce to the case $G=T, \rho: T \rightarrow D(V)=D\left(\oplus K \cdot v_{i}\right)$ where $\left\{v_{1}, \ldots, v_{n}\right\} \subseteq V$ is a diagonal basis. Write $V=\oplus_{\alpha \in \Phi(\rho)} V_{\alpha}$ where each $V_{\alpha}$ is a sum of $K \cdot v_{i}$ 's. Now $K[D(V)]=$ $K\left[x_{1}, \ldots, x_{n}\right], n=\operatorname{dim} V$, where each $x_{i}$ is the character of $D(V)$ obtained by restricting the action to $K \cdot v_{i}$. It then follows easily that $\rho^{*}\left(x_{i}\right)=\alpha$ iff $v_{i} \in V_{\alpha}$. 
Furthermore, by definition of $\Phi(\rho)$, each $\alpha \in \Phi(\rho)$ is hit. Thus, we obtain

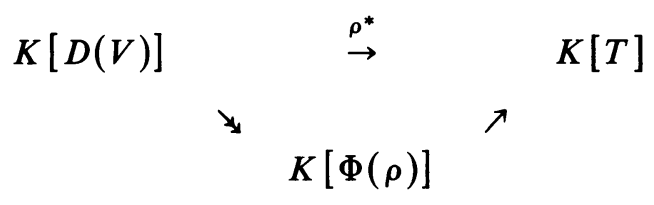

but the factorization is dual to

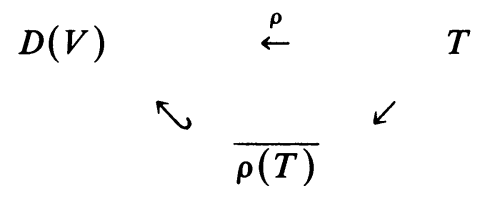

We conclude that $K[\overline{\rho(T)}]=K[\langle\Phi(\rho)\rangle]$. Thus, $X(\overline{\rho(T)})=\langle\Phi(\rho)\rangle$, since characters are linearly independent (the proof for monoids is the same as for groups $[2$, Lemma 16.1]).

Apply the lemma to the representation

$$
\rho: \mathrm{Sl}_{2}(K) \times K^{*} \rightarrow \mathrm{Gl}_{t}(K)
$$

of (1) and get $X(\rho(T)) \cong X(r)$. Thus, let $E^{\prime}=\overline{\rho\left(\mathrm{Sl}_{2}(K) \times K^{*}\right)} \subseteq \operatorname{End}_{K}\left(K^{t}\right)$ and then define

$$
E_{r}=\text { the normalization of } E^{\prime} \text {. }
$$

It is clear that $E$ is a normal monoid with group $G=\mathrm{Sl}_{2}(K) \times K^{*}$. Notice, as well, that $\bar{T}$ of $E_{r}$ is isomorphic to $\bar{T}^{\prime}$ of $E^{\prime}$ since under the normalization map

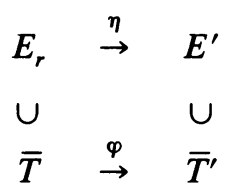

$\varphi$ is birational since $\left.\eta\right|_{G}$ is an isomorphism, and finite since $\eta$ is finite. Thus $\varphi$ is an isomorphism, since by construction and $4.1, \bar{T}^{\prime}$ is normal.

Let us summarize these results.

4.2. Proposition. Let $G=\mathrm{Sl}_{2}(K) \times K^{*}$ with maximal torus $T \subseteq G$ and let $X(r)=\{(k, l) \in \mathbf{Z} \oplus \mathbf{Z}|| k / l \mid \leqslant r\} \cup\{(0,0)\}$ for $r \in \mathbf{Q}^{+}$. Then there exists a normal algebraic monoid $E_{r}$ with 0 and unit group $G$ such that $X(\bar{T})=X(r)$.

It thus remains for us to construct the corresponding monoids for the groups $\mathrm{Gl}_{2}(K)$ and $\mathrm{PGl}_{2}(K) \times K^{*}$. The procedure is straightforward so it suffices to sketch the main points and leave the details to the reader.

Consider the following subgroups of $G=\mathrm{Sl}_{2}(K) \times K^{*}$.

$$
\begin{aligned}
& L=\left\{\left(\left[\begin{array}{cc}
-1 & 0 \\
0 & -1
\end{array}\right],-1\right),\left(\left[\begin{array}{ll}
1 & 0 \\
0 & 1
\end{array}\right], 1\right)\right\}, \\
& M=\left\{\left(\left[\begin{array}{cc}
-1 & 0 \\
0 & -1
\end{array}\right], 1\right),\left(\left[\begin{array}{ll}
1 & 0 \\
0 & 1
\end{array}\right], 1\right)\right\} .
\end{aligned}
$$


Plainly, $G / L \cong \mathrm{Gl}_{2}(K)$ and $G / M \cong \mathrm{PGl}_{2}(K) \times K^{*}$. Let $\pi: G \rightarrow \mathrm{Gl}_{2}(K)$ and $\theta$ : $G \rightarrow \mathrm{PGl}_{2}(K) \times K^{*}$ be the associated quotient morphisms.

Our strategy then is to construct the monoids with unit group $\mathrm{Gl}_{2}(K)$ and $\mathrm{PGl}_{2}(K) \times K^{*}$ as quotients of monoids with group $\mathrm{Sl}_{2}(K) \times K^{*}$.

Let $E_{r}$ be as in 4.2. Then we have group actions

$$
L \times E_{r} \rightarrow E_{r} \text { and } M \times E_{r} \rightarrow E_{r}
$$

defined by $(\alpha, x) \rightarrow \alpha \cdot x$ in each case.

4.3. Definition. $\bar{E}_{r}=E_{r} / L, E_{r}^{\prime}=E_{2 r} / M$.

Since $L$ and $M$ are finite $D$-groups [18, \$2.2] it follows from [16, Lemma 2.2] that $\bar{E}_{r}$ and $E_{r}^{\prime}$ are normal algebraic varieties. To prove that they are algebraic monoids, consider the following extension problem.

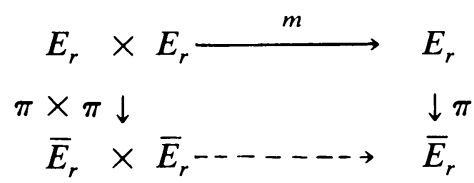

Since $\pi \times \pi$ is a quotient morphism, it suffices to prove that $\pi \circ m$ is constant on the fibres of $\pi \times \pi$.

But if $(g, h) \in L \times L$ and $(x, y) \in E_{r} \times E_{r}$ then

$$
\begin{aligned}
\pi \circ m(g x, h y) & =\pi(g x h y)=\pi(g h \cdot x y) \quad(L \text { is central }) \\
& =\pi(x y)=\pi \circ m(x, y)
\end{aligned}
$$

Thus, the dotted arrow can be filled in uniquely. Similarly, each $E_{r}^{\prime}$ is an algebraic monoid with group $\mathrm{PGl}_{2}(K) \times K^{*}$.

4.4. Proposition. Let $E=\bar{E}_{r}$ or $E_{r}^{\prime}$ be as in 4.3 and let $\bar{T} \subseteq E$ be the closure of $a$ maximal torus.

(a) If $G=\mathrm{PGl}_{2}(K) \times K^{*}$ then

$$
X(\bar{T})=\left\{(m, n) \in \mathbf{Z}^{2}|| m / n \mid \leqslant r\right\} \cup\{(0,0)\} .
$$

(b) If $G=\mathrm{Gl}_{2}(K)$ then

$$
X(\bar{T}):=Y(r)=\left\{(m, n) \in \mathbf{Z}^{2}|| \frac{m-n}{m+n} \mid \leqslant r\right\} \cup\{(0,0)\} .
$$

Proof. We have the quotient morphisms

$$
\pi: \mathrm{Sl}_{2}(K) \times K^{*} \rightarrow \mathrm{Gl}_{2}(K) \text { and } \theta: \mathrm{Sl}_{2}(K) \times K^{*} \rightarrow \mathrm{PGl}_{2}(K) \times K^{*}
$$

with $\operatorname{Ker} \pi=L$ and $\operatorname{Ker} \theta=M$. Let $T \subseteq \operatorname{Sl}_{2}(K) \times K^{*}$ be a maximal torus with $X(T)=\mathbf{Z}^{2}$.

(a) It follows from the definition of $M$ that

$$
X(T / M)=\{(m, n) \in X(T)|2| n\} .
$$

Thus, since $X(\bar{T} / M)=X(\bar{T}) \cap X(T / M)$,

$$
\begin{aligned}
X(\bar{T} / M) & =\{(m, n) \in X(T)|2| n,|n / m| \leqslant 2 r\} \quad\left[\text { recall } E_{r}^{\prime}=E_{2 r} / M\right] \\
& \cong\left\{(m, n) \in \mathbf{Z}^{2}|| n / m \mid \leqslant r\right\}
\end{aligned}
$$

under the $\operatorname{map}(m, n) \mapsto(m, n / 2)$. 
(b) It follows from the definition of $L$ that

$$
X(T / L)=\{(m, n) \in X(T)|2| m+n\} .
$$

Hence

$$
X(\bar{T} / L)=\{(m, n) \in X(T)|2| m+n,|n / m| \leqslant r\}
$$

under the map

$$
(m, n) \mapsto\left(\frac{m-n}{2}, \frac{m+n}{2}\right) .
$$

This completes the construction of the normal monoids with unit group $\mathrm{Sl}_{2}(K) \times$ $K^{*}, \mathrm{Gl}_{2}(K)$ and $\mathrm{PGl}_{2}(K) \times K^{*}$.

We now summarize the numerical information thus far achieved in the classification.

Let $E$ be an algebraic monoid with unit group $G$ as above and maximal torus $T \subseteq G$. Let $X=X(T), \Phi \subseteq X$ be the roots and $F=\left\{F_{1}, F_{2}\right\} \subseteq X$ the fundamental generators (as defined at the beginning of this section). Let $\langle x, y\rangle$ denote the free abelian group on the generators $x$ and $y$.

The polyhedral root system of $E$ is the triple $(X, \Phi, F)$. What follows is a complete list, for each group $G$, of the polyhedral root systems of monoids with group $G$ (this will follow from the results of §5). Although the complete list is not needed for this paper, we include it in this natural setting for future reference.

1.5. Polyhedral root systems.

$$
\begin{aligned}
& \frac{G=\mathrm{Sl}_{2}(K) \times K^{*}}{X=\langle x, y\rangle,} \\
& \boldsymbol{\Phi}=\{2 y,-2 y\}, \quad m, n>0, \\
& F=\{m x+n y, m x-n y\}, \quad(m, n)=1 \text {. } \\
& \frac{G=\mathrm{Gl}_{2}(K)}{X=\langle x, y\rangle,} \\
& \Phi=\{x-y, y-x\}, \quad m>|n| \geqslant 0, \\
& F=\{m x+n y, m y+n x\}, \quad(m, n)=1 . \\
& \frac{G=\mathrm{PGl}_{2}(K) \times K^{*}}{X=\langle x, y\rangle,} \\
& \boldsymbol{\Phi}=\{y,-y\}, \quad m, n>0, \\
& F=\{m x+n y, m x-n y\}, \quad(m, n)=1 .
\end{aligned}
$$

5. Uniqueness. The purpose of this section is to demonstrate that the monoids constructed in $\$ 4$ exhaust the possibilities for irreducible normal algebraic monoids with 0 and unit group $\mathrm{Sl}_{2}(K) \times K^{*}, \mathrm{Gl}_{2}(K)$ or $\mathrm{PGl}_{2}(K) \times K^{*}$.

Since the proofs in each case are so similar, we shall make the standing assumption that all significant results are to be proved for $\mathrm{Sl}_{2}(K) \times K^{*}$ and merely stated for the other two groups. 
To fix notation, let $E$ be a normal, irreducible algebraic monoid with 0 and unit group $\mathrm{Sl}_{2}(K) \times K^{*}$. Let $\bar{T} \subseteq E$ be the closure in $E$ of a maximal torus $T$ of $G=\mathrm{Sl}_{2}(K) \times K^{*}$.

Let $\eta: Z \rightarrow \bar{T}$ denote the normalization of the algebraic monoid $\bar{T}$. By equation (1) of $\$ 4$ (and the equation preceding it) it is clear that $Z \cong \bar{T}(r)$ for some $r \in \mathbf{Q}^{+}$.

Consider then, $E_{r}$ as in 4.2. If we let $j: G \rightarrow E$ be the inclusion, then an inclusion $i: G \rightarrow E_{r}$ can be chosen such that the following diagram commutes:

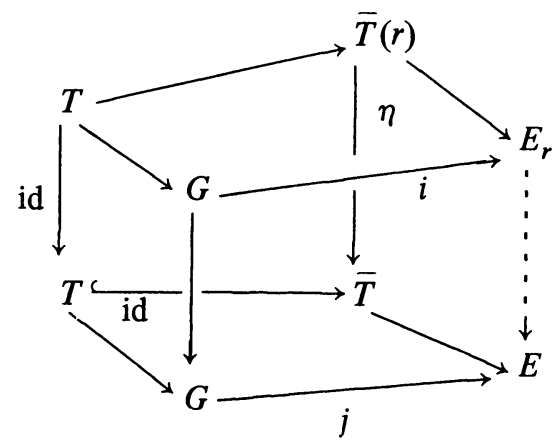

In particular, $\left.\eta\right|_{T}=\left.j\right|_{T}$.

5.1. LEMMA. Consider the diagram above. Then the dotted arrow can be filled in uniquely with an isomorphism of algebraic monoids $\varphi: E_{r} \rightarrow E$. The same results hold for the other two groups.

Proof. We have morphisms

$$
j: G\left(E_{r}\right) \rightarrow E \text { and } \eta: \bar{T}(r) \rightarrow E
$$

such that $\left.j\right|_{T}=\left.\eta\right|_{T}$. By 3.3 , there exists a unique morphism $\varphi: E_{r} \rightarrow E$ extending both $j$ and $\eta$. Let $S=\left\{x \in E \mid \operatorname{dim} \varphi^{-1}(x)>0\right\}$. By [2, Proposition 4.4] $S \subseteq E$ is closed. Also $G \cdot S \cdot G \subseteq S$ by homogeneity. Hence, $E \cdot S \cdot E \subseteq S$ as well because $G \subseteq E$ is dense. We thus conclude that if $S \neq \varnothing$ then $0 \in S$. We show $0 \notin S$.

Suppose $\varphi(x)=0, x \in E_{r}$. Since $E_{r}$ is regular [14], there exists $g, h \in G\left(E_{r}\right)$ such that $g x h \in I(\bar{T}(r))=\{1, e, f, 0\}$. Thus, $g x h=0$ since $\left.\varphi\right|_{\bar{T}(r)}$ is finite and sends only the 0 element to 0 . Hence, $\varphi^{-1}(0)=\{0\}$.

By definition of $S, \varphi$ is thus a finite to one morphism, yet by construction it is birational. Thus, by ZMT $\varphi$ is an open embedding. By [12], every idempotent of $E$ is conjugate to one of $\bar{T}$, and by construction each of these is in the image of $\varphi$. It follows that all idempotents of $E$ are in the image of $\varphi$. Thus $\varphi$ is also surjective since in a regular, irreducible monoid $E, G \cdot I(E) \cdot G=E$.

5.2. COROllary. Let $E$ be as above. Then $\bar{T} \subseteq E$ is a normal variety.

Proof. By 5.1, $\bar{T} \cong \bar{T}(r)$ which is normal by construction (see [3, Chapter 1] for more details on normal monoids associated with polyhedra).

5.3. Classification Theorem. Let $G$ be one of the groups $\mathrm{Sl}_{2}(K) \times K^{*}, \mathrm{Gl}_{2}(K)$ or $\mathrm{PGl}_{2}(K) \times K^{*}$ and let $\mathscr{E}(G)$ denote the set of isomorphy classes of irreducible, 
normal monoids with zero and unit group $G$. There is a canonical one-to-one correspondence, $\mathscr{E}(G) \cong \mathbf{Q}^{+}$.

Proof. This is mostly summary. By the results of $\S 4$, there exists, for each $r \in \mathbf{Q}^{+}$, a monoid $E=E_{r}$ with

$$
X(\bar{T}) \cong X(r)=\{(m, n)|| m / n \mid \leqslant r\} \cup\{(0,0)\} .
$$

Conversely, by Lemma 5.1, any $E \in \mathscr{E}(G)$ is isomorphic to $E_{r}$ for some $r \in \mathbf{Q}^{+}$. The result follows since $E_{r} \cong E_{s}$ implies $\bar{T}(r) \cong \bar{T}(s)$. But then $r=s$.

REMARKS. (i) For the monoids with group $\mathrm{PGl}_{2}(K) \times K^{*}, X(\bar{T})$ of $E_{r}$ is isomorphic to $X(r)$. (It is the placement of $\Phi$ in $X(T)$ that distinguishes $\mathscr{E}\left(\operatorname{Sl}_{2}(K) \times K^{*}\right)$ from $\mathscr{E}\left(\mathrm{PGl}_{2}(K) \times K^{*}\right)$ numerically (see 4.5$\left.)\right)$.

(ii) For the monoids with group $\mathrm{Gl}_{2}(K), X(\bar{T})$ of $E_{r}$ is isomorphic to

$$
Y(r)=\left\{(m, n) \in \mathbf{Z}^{2}|| \frac{m-n}{m+n} \mid \leqslant r\right\} \cup\{(0,0)\} .
$$

The remainder of this section is devoted to the case of normal monoids without a zero element. It turns out that there is only one such $E$ with $G \varsubsetneqq E$, for each group $G$.

Let $E$ be irreducible and normal with $G=\mathrm{Sl}_{2}(K) \times K^{*}$. We assume that $G \subsetneq E$, and also that no element $x$ of $E$ satisfies $x \cdot E=\{x\}=E \cdot x$. Let $\bar{T} \subseteq E$ be the closure in $E$ of a maximal torus $T$ of $G$. If $e \in \bar{T}$ is the minimal idempotent then $e$ is fixed under the action of $N_{G}(T)$, the normalizer of $T$ in $G$. By [9, Theorem 2.3], $e \in \bar{S}$, where $S \subseteq G$ is the identity component of the center of $G$. Hence, $\operatorname{dim}(e \cdot T)$ $>0$ since otherwise $e \cdot T=\{e\}$ for all maximal tori $T$ of $G$ ( $e$ is central and all maximal tori are conjugate). By [2, Theorem 22.2] or direct computation, $\cup T \subseteq G$ is dense. So we would then have $e G=G e=\{e\}$, contradicting the fact that $e$ is not the zero.

Consider $\bar{S} \subseteq E$ and let $G^{\prime}=(G, G) \subseteq G$. Define

$$
m: G^{\prime} \times \bar{S} \rightarrow E, \quad(g, t) \mapsto g \cdot t .
$$

5.4. Proposition. $m: G^{\prime} \times \bar{S} \rightarrow E$ is an isomorphism of algebraic monoids.

Proof. $m$ is birational since $S=\{1\} \times K^{*}$ and $G^{\prime}=\mathrm{Sl}_{2}(K) \times\{1\}$. From [6, Theorem 3.17], it follows that $I(\bar{T})=\{1, e\}$. So $I(E)=\{1, e\}$ since $e$ is central and by [12] every idempotent is conjugate to one in $\bar{T}$. Hence $m$ is surjective, since by [14], $E$ is a regular monoid. But $m$ is also finite to one since $E \backslash G=e \cdot G^{\prime}$, and so $\operatorname{dim}\left(e G^{\prime}\right)=\operatorname{dim}(E \backslash G)=\operatorname{dim} E-1=\operatorname{dim} G^{\prime}$. We thus conclude from ZMT, that $m$ is an isomorphism.

5.5. THEOREM. Let $G$ be one of the groups $\mathrm{Sl}_{2}(K) \times K^{*}, \mathrm{Gl}_{2}(K)$ or $\mathrm{PGl}_{2}(K) \times K^{*}$. In each case, there is exactly one isomorphy class of normal, irreducible monoid $E$ with no zero element and unit group $G \subsetneq E$.

(a) If $G=\mathrm{Sl}_{2}(K) \times K^{*}$ then $E=\mathrm{Sl}_{2}(K) \times K$.

(b) If $G=\mathrm{Gl}_{2}(K)$ then $E=\mathrm{Sl}_{2}(K) \times K /(x, t) \sim(-x,-t)$.

(c) If $G=\mathrm{PGl}_{2}(K) \times K^{*}$ then $E=\mathrm{PGl}_{2}(K) \times K$. 
6. Consequences of the classification. The classification in $\$ 5$ can be embellished to yield a better picture of the correspondence $\mathscr{E}(G) \cong \mathbf{Q}^{+}$of Theorem 5.3. It turns out that each $E_{r} \in \mathscr{E}(G)$ can be embedded in a commutative diagram of algebraic monoids

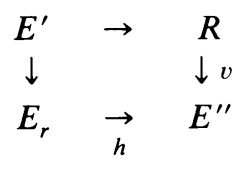

such that

(i) $R=\operatorname{End}_{K}\left(K^{2}\right)$;

(ii) $v$ and $h$ are finite and dominant;

(iii) $\operatorname{Ker}(h)$ and $\operatorname{Ker}(v)$ are $D$-groups;

(iv) the degrees of $v$ and $h$ are determined by $r$ and $G$.

The purpose of this section is to exhibit the details of that construction. Again we adopt the convention of proving the results for $\mathrm{Sl}_{2}(K) \times K^{*}$ and stating the analogues for the other two groups.

Let $E_{r} \in \mathscr{E}(G)$. By the results of $\S 5, \bar{T}$ of $E_{r}$ has

$$
X(\bar{T})=\left\{(m, n) \in \mathbf{Z}^{2}|| m / n \mid \leqslant r\right\} \cup\{(0,0)\} .
$$

Write $r=n / d$ where $(n, d)=1$.

Initially, our procedure is to construct morphisms

$$
v_{n}: E_{\alpha} \rightarrow E_{r} \text { and } h_{d}: E_{\alpha} \rightarrow R=\operatorname{End}_{K}\left(K^{2}\right)
$$

with the desired properties, where $\alpha=1 / d$. Define

$$
\psi_{n}: \mathrm{Sl}_{2}(K) \times K^{*} \rightarrow \mathrm{Sl}_{2}(K) \times K^{*}, \quad \psi_{n}(x, t)=\left(x, t^{n}\right) .
$$

The induced map on characters of respective maximal tori is

$$
\varphi: \mathbf{Z}^{2} \rightarrow \mathbf{Z}^{2}, \quad \varphi(k, l)=(k, n \cdot l) .
$$

But $\varphi(X(r)) \subseteq X(\alpha)$ since if $|k / l| \leqslant r$ then $|k / n l|=n^{-1}|k / l| \leqslant r / n=\alpha$. But $\varphi$ dualizes to give a morphism $\bar{\varphi}: \bar{T}(\alpha) \rightarrow \bar{T}(r)$ such that $\left.\varphi\right|_{T}=\left.\psi_{n}\right|_{T}$. Applying 3.3 yields a morphism $v: E_{\alpha} \rightarrow E_{r}$ such that $\left.v\right|_{G}=\psi_{n}$. We notice also that $\operatorname{Ker}(v)=$ $\left\{(1, t) \in \mathrm{Sl}_{2}(k) \times k^{*} \mid t^{n}=1\right\}$ is a finite $D$-group. In particular, degree $(v)=n$.

Similarly, one constructs a morphism $\eta_{d}: E_{\alpha} \rightarrow R$ of degree $2 d$ with finite $D$-group kernel. In this case, we start with the morphism

$$
\beta: \mathrm{Sl}_{2}(K) \times K^{*} \rightarrow \mathrm{Gl}_{2}(K), \quad \beta(x, t)=x \cdot\left[\begin{array}{cc}
t^{d} & 0 \\
0 & t^{d}
\end{array}\right] .
$$

The details are left to the reader.

6.1. TheOREM. Let $G=\mathrm{Sl}_{2}(K) \times K^{*}, \mathrm{Gl}_{2}(K)$ or $\mathrm{PGl}_{2}(K) \times K^{*}$ and let $E_{r} \in$ $\mathscr{E}(G)$. There exists a commutative diagram of algebraic monoids

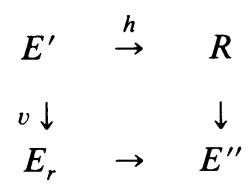


such that

(a) $R=\operatorname{End}_{K}\left(K^{2}\right)$;

(b) the kernel of each morphism is a finite D-group;

(c) $\operatorname{Ker}(h) \cap \operatorname{Ker}(v)=\{1\}$;

(d) if $G=\mathrm{Sl}_{2}(K) \times K^{*}$ then $r=2 \cdot \operatorname{deg}(v) / \operatorname{deg}(h)$;

if $G=\mathrm{Gl}_{2}(K)$ then $r=\operatorname{deg}(v) / \operatorname{deg}(h)$;

if $G=\mathrm{PGl}_{2}(K) \times K^{*}$ then $r=\operatorname{deg}(v) / 2 \cdot \operatorname{deg}(h)$.

Proof. By the previous discussion, we already have the appropriate $h$ and $v$ with $E^{\prime}=E_{\alpha}$. We must find $E^{\prime}$ with the desired properties.

Let $H=v(\operatorname{Ker}(h)) \subseteq E$ and define $E^{\prime \prime}=E / H$. Using the argument immediately following 4.2, it follows that $E^{\prime \prime}$ is a normal algebraic monoid and the quotient morphism $\pi: E \rightarrow E^{\prime \prime}$ is a morphism of algebraic monoids.

We are thus reduced to the following factorization problem.

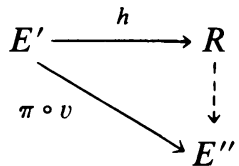

From the definition of $h$, it follows that $\eta: E^{\prime} / \operatorname{Ker}(h) \rightarrow R$ is a finite, birational morphism of normal, algebraic monoids. Thus, by ZMT, $\eta$ is an isomorphism. But now we can use the universal property of $h$ since $\pi \circ v$ vanishes on $\operatorname{Ker}(h)$. This concludes the proof.

\section{REFERENCES}

1. F. Grosshans, Observable groups and Hilbert's fourteenth problem, Amer. J. Math. 95 (1973), 229-253.

2. J. E. Humphreys, Linear algebraic groups, 2nd ed., Graduate Texts in Math., vol. 21, Springer Verlag, New York, 1981.

3. G. Kempf et al., Toroidal embeddings. I, Lecture Notes in Math., vol. 339, Springer Verlag, New York, 1973.

4. T. Oda, Torus embeddings and applications, TATA Inst. Fund. Res., Lectures on Math. and Phys., 57, Springer Verlag, New York, 1978.

5. M. S. Putcha, On linear algebraic semigroups, Trans. Amer. Math. Soc. 259 (1980), 457-469.

6. __ On linear algebraic semigroups. II, Trans. Amer. Math. Soc. 259 (1980), 471-491.

7. __ On linear algebraic semigroups. III, Internat. J. Math. and Math. Sci. 4 (1981), 667-690.

8. _ Linear algebraic semigroups, Semigroup Forum 22 (1981), 287-309.

9. __ A semigroup approach to linear algebraic groups, J. Algebra 80 (1983), 164-185.

10. The group of units of a connected algebraic monoid, Linear and Multilinear Algebra 12 (1982), 37-50.

11. __ Reductive groups and regular monoids, Semigroup Forum (to appear).

12. Green's relations on a connected algebraic monoid, Linear and Multilinear Algebra 12 (1982), 205-214.

13. L. Renner, Algebraic monoids, Thesis, Univ. of British Columbia, Vancouver, 1982.

14. , Reductive monoids are von Neumann regular, J. Algebra (to appear).

15. Quasi-affine algebraic monoids, Semigroup Forum (to appear).

16. Cohen-Macaulay algebraic monoids, Proc. Amer. Math. Soc. 89 (1983), 574-578.

17. I. Shafarevich, Basic algebraic geometry, Springer Study Edition, Springer Verlag, New York, 1977.

18. W. C. Waterhouse, Introduction to affine group schemes, Graduate Texts in Math., vol. 66, Springer Verlag, New York, 1979.

19. The unit groups of affine algebraic monoids, Proc. Amer. Math. Soc. 85 (1982), 506-508.

Department of Mathematics, York University, Downsview, Ontario, Canada 